\title{
CONSTRUCCIÓN Y VALIDACIÓN DE UN CUESTIONARIO SOBRE LOS HÁBITOS DE CONSUMO DE VIDEOJUEGOS EN PREADOLESCENTES
}

\author{
CONSTRUCTION AND VALIDATION OF A QUESTIONNAIRE ON VIDEO \\ GAME CONSUMPTION HABITS IN PRE-TEENS
}

\author{
Fernando López Becerra \\ f.lopez@ua.es \\ Universidad de Alicante
}

\begin{abstract}
RESUMEN
La identificación de los hábitos de consumo de videojuegos es una de las dimensiones a tener en cuenta de cara entender el contexto psicosocial y educativo los jóvenes. La bibliografía revisada en español ha utilizado habitualmente instrumentos de medida ad hoc, de cuestionable rigurosidad. Presentamos un cuestionario para identificar los hábitos de consumo de videojuegos entre los preadolescentes. El instrumento fue aplicado a 316 alumnos de tercer ciclo de Educación Primaria. Los resultados muestran una elevada fiabilidad (Alfa de Cronbach .915), y la existencia de 4 factores. En conclusión, se trata de un instrumento útil para evaluar los hábitos de consumo de videojuegos por parte de los preadolescentes.
\end{abstract}

PALABRAS CLAVE: Videojuegos, educación, tecnología de la información, cuestionario, adolescencia, ocio, medios audiovisuales, juegos.

ABSTRACT: Identifying video game habits is one of the dimensions to be taken into account for understanding the psychosocial context and youth education. The reviewed literature in Spanish is commonly used ad hoc measuring instruments, of questionable rigor. We present a questionnaire to identify the video game habits among preteens. The instrument was administered to 316 students of the third cycle of Primary Education. The results show high reliability (Cronbach's alpha .915), and the existence of 4 factors. In conclusion, it is a useful tool for assessing the consumption habits of video games by preteens.

KEYWORDS: Video games, education, information technology, questionnaire, adolescence, leisure, audiovisual media, games. 


\section{INTRODUCCIÓN}

En las tres últimas décadas se han producido grandes avances en nuestra sociedad. La mayoría de estos cambios están relacionados con el mundo audiovisual, el cual ha invadido casi todas las facetas de nuestra vida. Las nuevas tecnologías han traído progreso y han marcado, en muchos aspectos, un nuevo estilo de vida, así como una nueva manera de diversión. Los jóvenes se han adaptado rápidamente a la nueva realidad que les ofrecen los videojuegos, gracias, entre otros, a la estética, la jugabilidad y el entretenimiento que ofrecen.

La Asociación Española de Distribuidores y Editores de Software de Entretenimiento (ADESE) señala en sus resultados del año 2009 que la venta de software y hardware relacionado con el videojuego superó en cuota de mercado a lo registrado por la taquilla de cine, venta de DVD y música grabada, acaparando el 53\% del mercado total (ADESE, 2009a). En un estudio sobre hábitos de consumo realizado por esta misma asociación a personas de entre 7 y 34 años $(n=704)$ los resultados mostraron que alrededor de un $75 \%$ de los encuestados eran usuarios de videojuegos y entre un $10-15 \%$ jugaban todos los días, resultados que mostraban una clara tendencia al incremento de videojugadores en comparación informes anteriores (ADESE, 2009b). Aunque el número de jugadores mayores de 30 años ha aumentado en los últimos años, la mayoría de los "heavy players» (personas que dedican más de 2 horas a la semana a los videojuegos) son individuos en etapa adolescente 0 preadolescente (ADESE, 2009b).

La opinión de la comunidad científica sobre este fenómeno ha sido muy dispar. Muchas investigaciones realizadas sobre la influencia de los videojuegos se han centrado en los efectos negativos de los videojuegos violentos. Otras, en cambio, han revisado sus potencialidades, como por ejemplo la mejoría en las habilidades de atención visual gracias a los videojuegos de acción (Green y Bavelier, 2003; Okagaki y Frensch, 1994). Por su parte, en el ámbito educativo, algunas investigaciones han encontrado que los juegos y el software educativos son herramientas eficaces de enseñanza (Murphy, Penuel, Means, Korbak, y Whaley, 2001); del mismo modo que también se ha correlacionado negativamente el tiempo dedicado a los videojuegos con el rendimiento académico (Anderson y Dill, 2000; Anderson, Gentile, y Buckley, 2007).

Se hace patente que la tradicional dicotomía «bueno-malo» no tiene cabida en este marco de investigación, por lo que resulta inapropiado afirmar que «los videojuegos son malos para los niños», o viceversa, ya que hay efectos circunstanciales y coyunturales que ayudan a definir su naturaleza e idiosincrasia. En este sentido, Gentile y colaboradores (Gentile y Stone, 2005; Khoo y Gentile, 2007; Stone y Gentile, 2008) han sugerido que hay al menos cinco dimensiones que pueden influir en los efectos de los videojuegos: cantidad, contenido, contexto, estructura y mecánica.

El estudio de los hábitos de consumo de videojuegos se ha convertido en una línea de investigación prioritaria en la actualidad, como lo demuestra la ingente aparición de publicaciones recientes sobre el tema. Sin embargo, y aun teniendo una trayectoria histórica de más de veinticinco años, presenta lagunas importantes y opiniones contrapuestas entre los propios expertos. Es por ello que se demandan estudios e investigaciones diversas acerca 
de su uso, de las características significativas que poseen, del tipo de información y pautas conductuales que trasmiten, y de sus efectos entre la población juvenil. Por este motivo, resulta de especial relevancia la creación de instrumentos válidos y fiables que sirvan para averiguar y analizar el tipo de uso que realizan los adolescentes de los videojuegos. No obstante, en la mayoría de investigaciones que se han realizado en España en las que se ha necesitado conocer los hábitos de consumo de videojuegos, se han utilizado cuestionarios ad hoc, los cuales pueden no garantizar la fiabilidad y validez.

Sí que debemos destacar el cuestionario sobre uso y actitudes ante los videojuegos diseñado por Alfageme y Sánchez (2003), que ha sido utilizado por Sánchez, Alfageme y Serrano (2010); así como por de Díez et al. (2004), con las adaptaciones correspondientes. Por otro lado, de autores españoles también podemos destacar el trabajo de Tejeiro y Bersabé (2002), quienes crearon un cuestionario de nueve ítems para evaluar el uso de los videojuegos, basándose en los criterios del DSM-IV para la ludopatía y la drogadicción.

Dada la polémica y la desorientación que gira alrededor de la utilización de los videojuegos por parte de los niños y adolescentes, se ha planteado la creación de esta herramienta para conocer mejor el tipo y el grado de uso de los videojuegos. En este estudio se presenta un cuestionario para la evaluación de los hábitos de consumo de videojuegos diseñado específicamente para preadolescentes. Se presentan datos de la estructura factorial del instrumento, así como de la fiabilidad y la validez del mismo.

\section{Material y métodos}

\subsection{Participantes}

El tamaño de la muestra fue de $\mathrm{N}=316$ niños y niñas pertenecientes al tercer ciclo de Educación Primaria, es decir, a los niveles de 50 y 6으, del curso académico 2009/2010, de los cuales 170 son niños $(53,8 \%)$ y 146 son niñas $(46,2 \%)$. $146(46,2 \%)$ de los sujetos pertenecen a 5 o curso y $170(53,8 \%)$ a 6‥ Según la edad, hay 66 sujetos de 10 años, 138 de 11 años y 112 de 12 años.

\subsection{Instrumento}

El cuestionario a validar fue nombrado Cuestionario sobre hábitos de consumo de los videojuegos. Previamente a su diseño, se revisaron otros cuestionarios similares administrados en español; resultado del cual se llegó a la conclusión de que la mayoría de cuestionarios han sido realizados ad hoc, como por ejemplo el cuestionario de 37 ítems de López y León (2003); el Cuestionario de hábitos y preferencias de videojuegos compuesto por 45 preguntas de respuesta semiestructurada de Alonqueo y Rehbein (2008), el Cuestionario sobre uso y actitudes ante los videojuegos de Alfageme y Sánchez (2003), o los cuestionarios utilizados en tesis doctorales, como Sánchez (1997), Calvo (1997), o la reciente Encuesta sobre uso, características y preferencias con los videojuegos de Llorca (2009). De toda la revisión realizada, sólo Alfageme y Sánchez (2003) realizan un análisis del instrumento utilizado, aunque no de forma exhaustiva, por lo que considero que en este ámbito ha habido múltiples acercamientos, pero con limitada rigurosidad. 
El cuestionario, además de los campos descriptivos para anotar la edad y el sexo de los alumnos, consiste en 24 preguntas, que se reparten de la siguiente manera: 19 ítems tipo escala Likert con 5 alternativas de respuesta que van desde "Nada de acuerdo» (1) hasta «Totalmente de acuerdo» (5); y 5 ítems con cinco alternativas de respuesta cada uno.

Las preguntas del cuestionario nos proporcionan información sobre los siguientes aspectos: grado de atracción por los videojuegos, relación de los videojuegos con otras actividades, interferencia de los videojuegos en el rendimiento académico, y grado de inquietud que generan los videojuegos.

El cuestionario puede consultarse en el Anexo 1 de la investigación.

\subsection{Procedimiento}

Los 6 centros que participaron en esta investigación pertenecen a 3 localidades de la provincia de Alicante. Los tutores de tercer ciclo de Primaria fueron instruidos, ya que participaron en la administración de los cuestionarios.

Junto a los cuestionarios se les entregó un documento donde se explicitaban los motivos de la investigación y las instrucciones a seguir. Para preservar su derecho a la intimidad, las hojas de registro eran anónimas. De igual modo, se les informó a todos los participantes del carácter voluntario del estudio.

\subsection{Análisis de los datos}

Se llevaron a cabo análisis factoriales exploratorios para el estudio de la dimensionalidad de la prueba. Un primer análisis factorial con el método de factores principales y con el método de rotación oblicua, y un segundo análisis factorial con el método de extracción de componentes principales y el de rotación de Varimax, con el objetivo de clarificar la estructura de los factores que se extraían del cuestionario.

Se calculó el coeficiente de fiabilidad del cuestionario mediante el Alpha de Cronbach total y de cada uno de los factores que se extrajeron con los análisis factoriales. El paquete estadístico utilizado fue el SPSS 17.0.

\section{RESULTADOS}

\subsection{Análisis factoriales}

Mediante el análisis factorial se extrajeron cuatro factores, utilizando como método de extracción el de ejes principales y rotación oblimin directo. Las altas correlaciones encontradas entre estos cuatro factores parecen indicar que el cuestionario podría medir una única dimensión: "grado de atracción y uso de los videojuegos», es decir, que se trataría de un test unidimensional (Tabla 1). No obstante, del cuestionario nos interesan los cuatro factores, ya que cada uno de ellos nos informa de un rasgo interesante para comprender el grado de incidencia de los videojuegos en la vida del niño. 
Tabla 1: Matriz de correlaciones de entre los factores

\begin{tabular}{lllll}
\hline Factor & 1 & 2 & 3 & 4 \\
\hline 1 & 1,000 & & & \\
\hline 2 &, 280 & 1,000 & & \\
\hline 3 &, 392 &, 249 & 1,000 & \\
\hline 4 &, 243 &, 361 &, 251 & 1,000 \\
\hline
\end{tabular}

Se intentó identificar los diferentes ámbitos que nos pueden ayudar a la hora de averiguar el verdadero hábito de consumo de videojuegos, y así discriminar a quién tiene un hábito de consumo compatible con otras actividades y quién no. Por ello se repitió el análisis factorial con la finalidad de obtener la estructura más clara posible. En este caso, se utilizó como método de extracción decomponentes principales y como método de rotación, ortogonal, el varimax. De este modo, se pudieron identificar, a partir de la matriz de componentes, 4 claros y diferenciados rasgos en el patrón de consumo. En la Tabla 2 se muestran las matrices de los 4 factores que se extrajeron de este análisis factorial.

Tabla 2: Matriz de componentes rotados ${ }^{\mathrm{a}}$

\begin{tabular}{|c|c|c|c|c|}
\hline & \multicolumn{4}{|c|}{ Componente } \\
\hline & Factor 1 & Factor 2 & Factor 3 & Factor 4 \\
\hline Item1 & ,685 & & & \\
\hline Item2 & 632 & ,410 & & \\
\hline Item3 & ,672 & & & \\
\hline Item4 & ,686 & & & \\
\hline Item5 & ,604 & & & \\
\hline Item6 & ,647 & & & \\
\hline Item7 & ,445 & ,511 & & \\
\hline Item8 & & ,720 & & \\
\hline Item9 & & ,745 & & \\
\hline Item10 & & ,518 & & \\
\hline Item11 & & 638 & & \\
\hline Item12 & & & ,730 & \\
\hline Item13 & & & ,463 & \\
\hline Item14 & & & ,560 & \\
\hline Item15 & & & ,576 & \\
\hline Item16 & & & 696 & \\
\hline Item17 & & & & 689 \\
\hline Item18 & & & & 746 \\
\hline
\end{tabular}




\begin{tabular}{ll}
\hline Item20,635 & \\
\hline Item21 ,522 ,521 \\
\hline Item22, 798 \\
\hline Item23 ,789 \\
\hline Item24,612
\end{tabular}

Método de extracción: Análisis de componentes principales.

Método de rotación: Normalización Varimax con

Kaiser.

a. La rotación ha convergido en 7 iteraciones.

Los 4 factores que hemos extraído mediante el proceso estadístico explican el 55,991 \% de la varianza total del cuestionario. La composición factorial nos confirma que el factor predominante del cuestionario es el primero, lo cual concuerda con las expectativas iniciales.

El primer factor está formado por los ítems 1, 2, 3, 4, 5, 6, 20, 21, 22, 23, 24. Explica el $35,637 \%$ de la varianza total del cuestionario. Nos informa sobre el los hábitos de uso y el grado de atracción que ejercen los videojuegos sobre el niño. A este factor se le denomina «Grado de atracción por los videojuegos».

El segundo factor está formado por los ítems 7, 8, 9, 10, 11. Explica el 10,043 \% de la varianza total del cuestionario. Nos informa sobre el grado de interferencia que tiene el acto de videojugar respecto a otras actividades no educativas. A este factor se le denomina «Interferencia de los videojuegos con otras actividades».

El tercer factor está formado por los ítems 12, 13, 14, 15, 16. Explica el 5,625 \% de la varianza total del cuestionario. Nos informa de la medida en que los niños son capaces de ingeniárselas para jugar, conseguir videojuegos, o buscar información sobre los videojuegos que más les pueden gustar. A este factor se le denomina «Nivel de inquietud respecto a los videojuegos».

El cuarto factor está formado por los ítems 17, 18, 19. Explica el 4,687 \% de la varianza total del cuestionario. Nos informa sobre el grado de interferencia que suponen los videojuegos en las actividades académicas. A este factor se le denomina «Interferencia de los videojuegos con las actividades académicas».

\subsection{Análisis de fiabilidad}

El Alpha de Cronbach que informa de la fiabilidad del cuestionario es de 0,915. El Alpha del factor 1 supera el 0,80 y el de los factores 2,3 , y 4 se sitúa entre 0,65 y 0,80. Los Alpha de Cronbach de cada uno de los factores se resumen en la Tabla 3.

Tabla 3: Coeficientes Alpha de Cronbach para los distintos factores

\begin{tabular}{l|l|l|l|l}
\hline Factor & 1 & 2 & 3 & 4 \\
\hline Alpha & 0,903 & 0,767 & 0,740 & 0,696 \\
\hline
\end{tabular}




\subsection{Análisis de frecuencias}

El análisis de frecuencias nos indica que prácticamente todos los alumnos juegan a los videojuegos, aunque cerca del $20 \%$ lo hace de manera esporádica o casi nunca. Un 17,41\% reconoce jugar a diario, aunque el grueso de la muestra juega los fines de semana.

Por otro lado, casi la mitad de los encuestados afirman jugar menos de 1 hora a la videoconsola, mientras que sólo el $7,28 \%$ lo hace durante 3 horas o más. El $6 \%$ afirma no jugar a la videoconsola. Estos datos dibujan un paisaje donde los niños juegan a la videoconsola de dos a cuatro días a la semana, manteniendo la actividad hasta dos horas.

\subsection{Relación entre los hábitos de consumo de videojuegos y el género}

Para comprobar la diferencia de hábitos de consumo entre género se ha aplicado la prueba $t$ de Student para muestras independientes para todos los factores. Los resultados indican que los chicos muestran un hábito de consumo de videojuegos estadísticamente significativo superior al de las chicas (Factor $1, t=10,942 ; p=, 000$ ). Además, los varones manifiestan un grado de interferencia de los videojuegos sobre otro tipo de actividades significativamente mayor que las féminas (Factor 2, $t=5,155 ; p=, 000$ ). Ellos también muestran un nivel de inquietud por los videojuegos significativamente superior que ellas (Factor $3 \mathrm{t}=9,270$; $p=, 000)$. Por último, los varones manifiestan un grado de interferencia de los videojuegos sobre las actividades académicas significativamente mayor que las féminas (Factor $4, t=$ 4,587; $p=, 000)$.

Respecto al número de horas que dedican al día a videojugar también encontramos diferencias estadísticamente significativas entre ellos y ellas $(t=7,078 ; p=, 000)$. Ellos dedican de, de media, de 1 a 2 horas al día, mientras que ellas juegan mayoritariamente menos de una hora diaria.

\subsection{Relación entre los hábitos de consumo de videojuegos y el curso académico}

Ninguno de los cuatro factores muestra cambios estadísticamente significativos en función del curso académico. Tanto los alumnos de 50 como los de 6ㅇ curso manifiestan unos hábitos de uso, grado de atracción e interferencia similares.

\section{DISCUSIÓN}

A día de hoy existe un debate sobre la nueva manera de ocio que suponen los videojuegos, siendo, en general, desfavorable la opinión pública hacia este tipo de entretenimiento. Este rechazo se produce porque en este ámbito predominan las creencias y tópicos basados en estereotipos, más que en observaciones directas y estudios científicos, tendencia que han advertido distintos autores en diferentes momentos de la historia de los videojuegos, como Estalló (1995), López y León (2003), o más recientemente Tejeiro, Pelegrina y Gómez (2009), quienes afirman que los prejuicios morales y el desconocimiento han estado implicados en las apreciaciones sobre los videojuegos desde que aparecieron. Esta situación pone de manifiesto la necesidad de seguir investigando y aclarar las dudas que suscitan este debate. 
Con la intención de profundizar sobre este tema ha sido creado el Cuestionario sobre hábitos de consumo de los videojuegos.

La validez de constructo del cuestionario, valorada a través del análisis factorial de las respuestas de los encuestados, demostró que la encuesta tenía 4 factores principales. La fiabilidad encontrada en cada uno de esos factores fue entre alta y muy alta.

El eje del cuestionario es muy motivador para el alumnado, lo cual produjo que los alumnos lo recibiesen con agrado y buena disposición. Esta característica favorece su administración, ya que los jóvenes muestran entusiasmo mientras lo cumplimentan.

Consideramos que el cuestionario se puede adaptar dependiendo del objetivo del estudio. En este caso nos hemos limitado a medir el grado y los hábitos de uso de los videojuegos, abandonando otros aspectos que también pueden ser influyentes para comprender la incidencia del fenómeno de los videojuegos entre los jóvenes. Se podrían introducir, por ejemplo, variables que examinen con exactitud el tipo de juego al que juegan los preadolescentes. Esta variable puede convertirse en el eje de futuras investigaciones, para así analizar las posibles relaciones entre el tipo de juego preferido, y otras variables como el rendimiento académico, agresividad, actitud ante la cultura, disposición hacia la actividad física, etc. De este modo se podría analizar si, igual que el espectador que suele visionar documentales de televisión no presenta las mismas características que quienes ven programas sensacionalistas, el adolescente que prefiere juegos con un alto nivel de exigencia cognitiva no compartirá las mismas características personales ni académicas que aquél que prefiere los juegos de habilidad óculo manual.

Respecto a los resultados descriptivos, el $94 \%$ de los alumnos encuestados manifestó jugar con videojuegos, aunque si nos ceñimos únicamente a quienes lo realizan con asiduidad, este porcentaje se reduce al $79,1 \%$. Esta cifra coincide con la obtenida por López y León (2003), quienes encontraron que el $77,29 \%$ de sus encuestados jugaban frecuentemente con videojuegos. Por otro lado, el hecho de que tan sólo el 1,90\% de los encuestados reconozca no haber jugado nunca a los videojuegos es coincidente con la investigación llevada a cabo por Llorca (2009), donde el 2,6\% de la muestra se encontraba en esta misma situación. Este dato confirma la generalización de los videojuegos dentro del ocio infantil, donde apenas dos de cada cien niños no ha jugado nunca a un videojuego.

Toda actividad es susceptible de convertirse en perniciosa si el número de horas que se le dedica es tan alto que se abandonan el resto de actividades. Es ahí cuando entra en juego el adulto, que es el responsable de controlar al infante, que todavía no es lo suficientemente maduro para comprender la trascendencia de sus actos.

Por tanto, creo que no debemos culpabilizar a los videojuegos por motivar, sino aprovechar esta característica para beneficiar a los niños. Por ello, considero que las investigaciones, a partir de ahora, deberían centrarse en averiguar las potencialidades de los videojuegos, más que valorar hasta qué punto son dañinos o perjudiciales.

El análisis de género nos muestra que los varones manifiestan un mayor grado de atracción por los videojuegos que las féminas. Puede que una razón de que las chicas tradicionalmente hayan jugado menos a los videojuegos sea porque los juegos hayan sido pensados para un imaginario masculino que dan respuesta a los deseos, afinidades y aficiones de los chicos, como afirman Díez et al. (2004). Estos resultados coinciden con la investigación de López y 
León (2003), en la que el 55'62\% de las chicas declaraba videojugar habitualmente, mientras que, en el caso de los chicos, el porcentaje subía hasta el $89^{\prime} 36 \%$. Por su parte, Lucas y Sherry (2004), confirmaron en su investigación que las mujeres jugaban menos que los hombres: el $54.6 \%$ de ellas y el $88.3 \%$ de ellos eran jugadores. Las mujeres dedicaban 4,25 horas de media a la semana, y ellos 11 horas de media. Además, tras la revisión que realizaron, Lucas y Sherry (2004) concluyeron que un hallazgo estable a lo largo de las últimas décadas en la investigación sobre los videojuegos es que son jugados mayoritariamente por hombres que por mujeres. No obstante, hay que señalar que en los últimos años la industria del videojuego se está implicando en la incorporación de la mujer al mundo de los videojuegos.

No se han encontrado grandes discrepancias entre los hábitos de consumo de videojuegos de los alumnos de quinto y sexto. En líneas generales, podemos afirmar que quien era videojugador en quinto, continúa siéndolo en sexto. Estos resultados permiten deducir que el patrón de consumo no varía significativamente en un año, aunque no sabemos si existe un cambio en cuanto a gustos y preferencias.

El desarrollo de este tipo de herramientas supone un paso más para comprender el alcance y la influencia que supone la irrupción de los videojuegos en nuestra sociedad. Quizás la próxima meta consista en identificar las diferencias que produce la práctica con los distintos tipos de videojuegos que existen en el mercado, ya que este tipo de producto es muy heterogéneo, y en demasiadas ocasiones se les engloba como un todo. De esta manera podríamos comprender mejor este fenómeno que se ha asentado como un hábito entre la juventud actual.

\section{REFERENCIAS}

ALFAGEME, B., \& SÁNCHEZ, P. (2003). Un instrumento para evaluar el uso y actitudes hacia los videojuegos. Píxel-Bit, 20, 17-32.

ALONQUEO, P., \& REHBEIN, L. (2008). Usuarios habituales de videojuegos: una aproximación inicial. Última Década, 29, 11-27.

ANDERSON, C. A., \& DILL, K. E. (2000). Video Games and Aggressive Thoughts, Feelings, and Behavior in Laboratory and in Life. Journal of Personality and Social Psychology, April (78), 4, 772-790.

ANDERSON, C. A., GENTILE, D. A., \& BUCKLEY, K. E. (2007). Violent video game effects on children and adolescents: Theory, research, and public policy. New York: Oxford University Press.

Asociación española de editores y distribuidores de software de entretenimiento (ADESE). (2009a). Resultados anuales 2009. (http://www.adese.es/pdf/Presentacion balance 2009.pdf) (25-03-2012).

Asociación española de editores y distribuidores de software de entretenimiento (ADESE). (2009b). Usos y hábitos de los videojugadores españoles. (http://www.adese.es/pdf/FaseUAadese122009.pdf) (25-03-2012). 
CALVO, A. (1997). Ocio en los noventa: los videojuegos. Estudio sobre la invidencia de los videojuegos en los jóvenes de Mallorca. Tesis Doctoral. Universitat de les Illes Balears.

DÍEZ, E. J. (2004). La diferencia sexual en el análisis de los videojuegos. CIDE, Instituto de la Mujer.

ESTALLÓ, J. A. (1995). Los videojuegos. Juicios y prejuicios. Barcelona: Planeta.

GENTILE, D. A., \& STONE, W. (2005). Violent video game effects on children and adolescents: A review of the literature. Minerva Pediatrica, 57, 337-358.

GREEN, S., \& BAVELIER, D. (2003). Action video game modifies visual selective attention. Nature, 423, 534-537.

KHOO, A., \& GENTILE, D. A. (2007). Problem-based learning in the world of games. En O. S. Tan \& D. Hung (Eds.), Problem-based learning and e-learning breakthroughs (pp. 97-129). Singapore: Thomson Publishing.

LLORCA, M. A. (2009). Hábitos y uso de los videojuegos en la comunicación visual: Influencia en la inteligencia espacial y el rendimiento escolar. Tesis Doctoral. Universidad de Granada.

LÓPEZ, M. J. \& LEÓN, R. (2003). Los adolescentes y los videojuegos. Apuntes de Psicología, $21,1$.

LUCAS, K. \& SHERRY, J. L. (2004). Sex differences in video game play: A communicationbased explanation. Communication Research, 31, 499-523.

MURPHY, R., PENUEL, W., MEANS, B., KORBAK, C., \& WHALEY, A. (2001). E-DESK: A review of recent evidence on the effectiveness of discrete educational software. Menlo Park, CA: SRI International. (http://ctl.sri.com/publications/downloads/Task3 FinalReport3.pdf) (25-032012).

OKAGAKI, L. \& FRENSCH, P. (1994). Effects of video game playing on measures of spatial perfomance: gender effects in late adolescence. Journal of Applied Development Psychology. Jan-Mar 15 (1) 33-58.

SÁNCHEZ, J. (1997). Valores, Estilos de vida y educación de los niños en relación al videojuego. Bases para un programa de acción. Tesis Doctoral. Universidad de Valencia.

SÁNCHEZ, P. A., ALFAGEME, M. B., \& SERRANO, F. J. (2010). Aspectos sociales de los videojuegos. Revista Latinoamericana de Tecnología Educativa RELATEC, 9 (1), 43-52.

STONE, W. \& GENTILE, D. A. (2008). The five dimensions of video game effects. Paper presented at the Annual Convention of the American Psychological Association, Boston.

TEJEIRO, R., \& BERSABÉ, R. (2002). Measuring problem video game playing in adolescents. Addiction, 97, 1601-1606.

TEJEIRO, R., PELEGRINA, M., \& GÓMEZ, J. L. (2009). Efectos psicosociales de los videojuegos. Comunicación, 7, Vol.1, 235-250. 


\section{Anexo 1: Cuestionario sobre hábitos de consumo de los videojuegos}

Estamos interesados en conocer tus hábitos de consumo de videojuegos. Cuando hablamos de videojuegos, incluimos los de consola y ordenador. Lee atentamente las oraciones del cuestionario. Rodea el número de la respuesta que quieres marcar con un bolígrafo.

Valoramos mucho tu sinceridad. No hay respuestas mejores ni peores. Tan sólo te pedimos que contestes sobre tus hábitos con respecto a los videojuegos. Si tienes alguna duda, levanta la mano y el profesor te atenderá. Muchas gracias por tu colaboración.

Curso .......... Edad ...........Chico/ Chica. Colegio:

\begin{tabular}{|c|c|c|c|c|c|c|}
\hline & & $\begin{array}{ll}\frac{0}{0} & \\
& \frac{0}{0} \\
\frac{\pi}{0} & \frac{0}{0} \\
\frac{\pi}{2} & \frac{\pi}{0} \\
\end{array}$ & 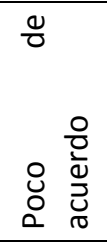 & 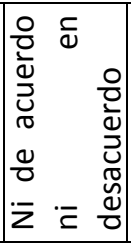 & 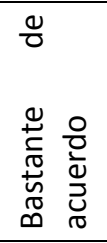 & 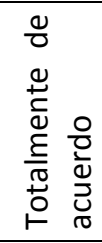 \\
\hline 1. & Me gusta jugar a los videojuegos. & 1 & 2 & 3 & 4 & 5 \\
\hline 2. & Juego habitualmente a los videojuegos. & 1 & 2 & 3 & 4 & 5 \\
\hline 3. & He jugado a muchos videojuegos. & 1 & 2 & 3 & 4 & 5 \\
\hline 4. & Conozco muchos videojuegos. & 1 & 2 & 3 & 4 & 5 \\
\hline 5. & Me considero bueno jugando a los videojuegos. & 1 & 2 & 3 & 4 & 5 \\
\hline 6. & Los videojuegos me parecen divertidos. & 1 & 2 & 3 & 4 & 5 \\
\hline 7. & Cuando juego a los videojuegos se me pasa el tiempo volando & 1 & 2 & 3 & 4 & 5 \\
\hline 8. & Dedico más tiempo a los videojuegos que jugar con mis amigos. & 1 & 2 & 3 & 4 & 5 \\
\hline 9. & Dedico más tiempo a jugar a los videojuegos que al deporte. & 1 & 2 & 3 & 4 & 5 \\
\hline 10. & Me acuesto tarde y me levanto temprano para seguir jugando. & 1 & 2 & 3 & 4 & 5 \\
\hline 11. & Dedico más tiempo a los videojuegos que a estar con mi familia. & 1 & 2 & 3 & 4 & 5 \\
\hline 12. & Busco información sobre videojuegos en revistas, TV o Internet. & 1 & 2 & 3 & 4 & 5 \\
\hline 13. & Me gusta competir a los videojuegos y ser el mejor. & 1 & 2 & 3 & 4 & 5 \\
\hline 14. & Ahorro mi dinero para gastarlo en videojuegos. & 1 & 2 & 3 & 4 & 5 \\
\hline 15. & Hablo con mis amigos de videojuegos. & 1 & 2 & 3 & 4 & 5 \\
\hline 16. & Siempre que veo una tienda de videojuegos entro. & 1 & 2 & 3 & 4 & 5 \\
\hline 17. & Antes de hacer los deberes juego a los videojuegos. & 1 & 2 & 3 & 4 & 5 \\
\hline 18. & $\begin{array}{l}\text { Dedico más tiempo a los videojuegos que a hacer las tareas del } \\
\text { cole. }\end{array}$ & 1 & 2 & 3 & 4 & 5 \\
\hline 19. & Olvido cosas importantes mientras juego (hacer los deberes...) & 1 & 2 & 3 & 4 & 5 \\
\hline
\end{tabular}

Escoge, de las siguientes preguntas, la respuesta que más se acerque a la realidad:

\begin{tabular}{|l|l|l|l|l|l|l|}
\hline 20. & Juego a los videojuegos desde hace: & Nunca & Meses & Un año & 2 o 3 años & +de 4 años \\
\hline 21. & Dedico a los videojuegos: & Nada & $\begin{array}{l}\text { Menos de 1 } \\
\text { hora al día }\end{array}$ & $\begin{array}{l}\text { De } 1 \text { a 2 } \\
\text { horas al día }\end{array}$ & $\begin{array}{l}\text { De } 2 \text { a } 3 \\
\text { horas al día }\end{array}$ & $\begin{array}{l}\text { Más de } 3 \\
\text { horas al día }\end{array}$ \\
\hline 22. & Número de videojuegos que conozco: & 0 & 1 o 2 & Hasta 10 & De 10 a 20 & + de 20 \\
\hline 23. & $\begin{array}{l}\text { Número de videojuegos que he } \\
\text { jugado: }\end{array}$ & 0 & 1 o 2 & Hasta 10 & De 10 a 20 & + de 20 \\
\hline 24. & Frecuencia a la que juego: & Nunca & $\begin{array}{l}\text { Alguna vez } \\
\text { al mes }\end{array}$ & $\begin{array}{l}\text { Fines de } \\
\text { semana }\end{array}$ & $\begin{array}{l}\text { Tres o } \\
\text { cuatro días }\end{array}$ & $\begin{array}{l}\text { Todos los } \\
\text { días }\end{array}$ \\
\hline
\end{tabular}


LÓPEZ, F. (2012). Construcción y validación de un cuestionario sobre los hábitos de consumo de videojuegos preadolescentes. EDUTEC, Revista Electrónica de Tecnología Educativa, 40. Recuperado el dd/mm/aa de http://edutec.rediris.es/Revelec2/Revelec40/contrusccion validacion cuestionario habitosconsumo videojuegos adolescentes.html 